\title{
Urinary Calcium to Creatinine Ratio in Preeclampsia - a Comparative Study
}

\author{
Authors

\section{Dr Anita $V^{1}$, Dr Adma Harshan $S^{2}$} \\ ${ }^{1}$ Assistant Professor, Department of Obstetrics and Gynaecology, Government Medical College Maneri \\ ${ }^{2}$ Associate Professor, Department of Obstetrics and Gynaecology, Government Medical College Maneri \\ Corresponding Author
}

Dr Anita V.

Email: anitaunnikrishnan@gmail.com

\begin{abstract}
Aim: An investigation To study whether urinary calcium and calcium to creatinine ratio are decreased in pregnant patients with preeclampsia.

Material \& Methods: The study was conducted in Sree Avittom Thirunal Hospital, Thiruvananthapuram, during a period of seven months. A total of 100 patients were included in the study out of which 50 were preeclamptic patients and (group 1) another 50 belonged to normo tensive group (group 2). The study group were all primi gravidas, in their third trimesters, on unrestricted diet and with no history of diabetes or renal diseases. Estimation of urinary calcium and creatinine was done at bio-chemistry laboratory of the same hospital. Relevant history like age, socio-economic status, mean arterial pressure, family history of hypertension and history of passive smoking were also collected. Data collected were entered into a master chart and statistical packages were used to compute significance.

Results: Urinary calcium to creatinine ratio was significantly reduced (tvalue 3.57, $p$ 0.0005) in preeclampsia patients as compared to normotensive group. Mean arterial pressure, family history of hypertension and history of passive smoking had no significant association with occurence of preeclampsia in pregnancy.

Conclusion: This study confirms hypo calciuria and decreased urinary calcium to creatinine ratio in preeclampic patients. The mechanism is not well established. The ratio can be used as a predictive tool for development of preeclampsia in pregnancy. Mean values of calcium excretion obtained in this study are lower than that in various other studies. This may be due to dietary deficiency of calcium.

Keywords- Preeclampsia, hypo calciuria, calcium to creatinine ratio, hyper tension.
\end{abstract}

\section{Introduction}

Hypertensive disorders are one of the most common medical complications during pregnancy. The incidence of hypertensive disorder is about $7-10 \%$. Despite numerous efforts at early diagnosis prevention and treatment of these disorders, they remain a major cause of adverse maternal and perinatal outcome. Once diagnosis of preeclampsia has been made, treatment options are limited. Much attention therefore has focused on preventive and predictive strategies. Several studies using measurement of 24 hour urinary calcium excretion 
have documented decreased excretion in women with preeclampsia. 24 hour urinary calcium excretion related well with calcium creatinine ratio of a single void urine sample. Calcium to creatinine ratio was also found to be a useful screening tool in predicting development of preeclampsia.

\section{Material and Methods}

The study population included 50 preeclampsia patients given as group 1 and group 2 consisted of 50. normotensive pregnant patients. All women were in their third trimesters and were on unrestricted diet. Primigravidas were included in the study. Women with diabetis and renal diseases were excluded from the study. Women were defined as belonging to preeclampsia group if their blood pressure were above $140 / 90 \mathrm{mmHg}$ on two occasions 6 hours apart and 2+ proteinuria in urine sample. Early morning sample of urine were collected Relevant history of the patient was recorded in the proforma for instance, age, socio economic status, number of months married, mean arterial pressure, family history of hypertension, history of passive smoking.

Quantitative determination of urinary calcium was performed by a colorimetric technique using cresophthalein dye which binds with calcium to produce a coloured complex measured at 565-578 $\mathrm{nm}$ on a semi auto analyser. Urinary creatinine is measured by Jaffe creation with alkaline picrate reagent.

Data collected were entered into a master sheet and necessary statistical tables and diagrams prepared. Equality of mean values were tested statistically using student's t test. Association between variables were tested using chi square tests. In order to find out the possible range in which $95 \%$ sample estimates lie, $95 \%$ confidence intervals were calculated. Invariably in all calculations, statistical packages were used.

\section{Results}

The study population of preeclamptic patients constituted Group 1 and normotensive pregnant patients constituted Group 2. The data was analysed and following observations were made.

\section{Urinary Calcium}

The entire document should be in Times New Roman of font size 10. Other font types may be used if needed for special purposes.

Table 1

\begin{tabular}{|l|l|l|l|}
\hline Group & Observations & Mean & SD \\
\hline Gp 1 & 50 & 4.834 & 0.822 \\
\hline Gp 2 & 50 & 7.520 & 0.725 \\
\hline
\end{tabular}

ie, There is significant difference in urinary calcium excretion between the 2 groups. In the normotensive group, mean urinary excretion was $7.52 \mathrm{mg} / \mathrm{dl}$ while it was $4.8 \mathrm{mg} / \mathrm{dl}$, in pre eclampsia group. Thus a significant reduction in calcium excretion was found in pre eclampsia.

\section{Urinary Creatinine Excretion}

In case of urinary creatinine levels, mean was 65.28 $\mathrm{mg} \%$ in pre eclampsia and $65.3 \mathrm{mg} \%$ in normotensive group. In this case, difference in mean values were not found to be significant i.e., there doesn't seem to be much difference in creatinine excretion in both these groups.

\section{Table 2}

\begin{tabular}{|l|l|l|l|l|}
\hline Group & Observations & Total & Mean & SD \\
\hline Gp 1 & 50 & 3264 & 65.280 & 42.56 \\
\hline Gp 2 & 50 & 3265 & 65.30 & 19.75 \\
\hline
\end{tabular}

\section{Urinary Calcium - Creatinine ratio}

Table 3

\begin{tabular}{|l|c|c|c|}
\hline Group & Observations & Mean & SD \\
\hline Gp 1 & 50 & 0.0925 & 0.047 \\
\hline Gp 2 & 50 & 0.125 & 0.037 \\
\hline
\end{tabular}

$\mathrm{t}$ value $-3.57, \mathrm{p}$ value 0.0005

Urinary calcium creatinine ratio showed significant reduction in pre eclampsia with mean being $0.09 \&$ 0.125 in normotensive. Since $24 \mathrm{hr}$ urinary calcium excretion correlates well with the $\mathrm{Ca} / \mathrm{Cr}$ ratio of early morning single urine specimen, this test can be used as a screening test for detecting patients prone for developing pre eclampsia and this test is less cumbersome too. 


\section{Family History of Hypertension}

Table 4

\begin{tabular}{|l|l|l|}
\hline Group & Gp 1 & Gp 2 \\
\hline Yes & 18 & 14 \\
\hline No & 32 & 36 \\
\hline
\end{tabular}

Chisquare $-0.41, \mathrm{p}$ value $-\mathrm{m} 0.520$

$36 \%$ in pre eclampsia group had positive family history of hypertension \& $28 \%$ in normotensive group. When these values were statistically computed, it was found to be not significant. In this study, family history of hypertension was not found to have much influence on development of pregnancy induced hypertension.

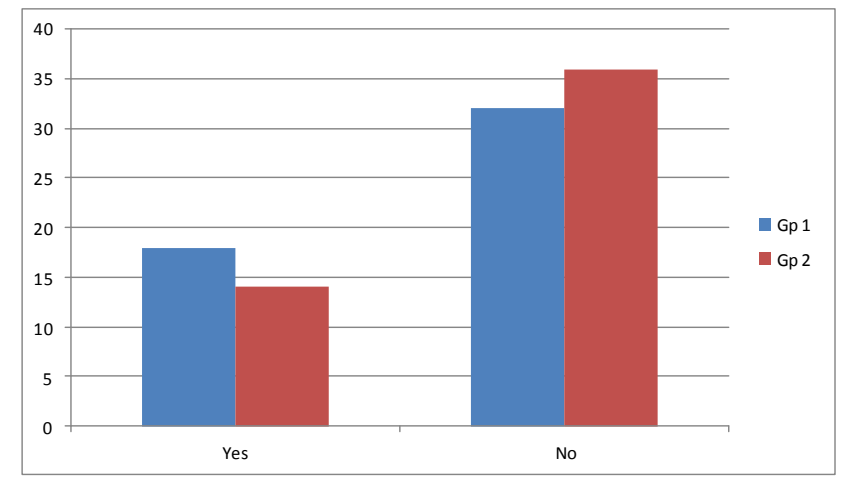

Fig. 1 Example of a High definition image

\section{History of Passive Smoking}

\section{Table 5}

\begin{tabular}{|l|c|c|}
\hline Group & Gp 1 & Gp 2 \\
\hline Yes & 19 & 23 \\
\hline No & 31 & 27 \\
\hline
\end{tabular}

Chisquare $-0.37, \mathrm{p}$ value -0.5432

$62 \%$ of pre eclampsia patients had no history of passive smoking while it was $54 \%$ in case of normotensives. No statistically significant relationship could be established between passive smoking \& pregnancy induced hypertension.

\section{Discussion}

In this study, urinary calcium to creatuinine ratio was found to be decreased in patients with pre eclampsia. Urinary calcium excretion in normal pregnancy is $350-620 \mathrm{mg} /$ day compared to $100-250$ $\mathrm{mg}$ /day in nonpregnant women, Excretion usually increases during each trimester, with maximum levels reached during the third trimester. Serum calcium levels in pre-eclampsia is similar to values in normotensives, but urinary calcium is markedly reduced. Calcium metabolism during pre-eclampsia is characterized by minor changes in serum levels of total and ionized calcium, but urinary calcium is markedly reduced. Calcium metabolism during pregnancy is characterized by minor changes in serum levels of total and ionized calcium, but urinary calcium excretion increases markedly during normal pregnancy.

The Mayan Indians of Gautemala have a low incidence of pre-eclampsia \& eclampsia ${ }^{(1)}$. The observation that the diet of these native people is selectively enriched with calcium because of their tradition of soaking coca in lime before cooking led to the hypothesis that hypertensive disorders of pregnancy may be related to calcium intake. Indeed epidemiologic studies in the early 1980's confirmed an inverse relationship between calcium intake and blood pressure during pregnancy.

Low calcium intake may cause high blood pressure by stimulating the release of parathyroid hormone and/or renin thereby increasing intra cellular calcium in vascular smooth muscle and causing vasoconstriction.

Alternatively, low calcium intake may effect vascular reactivity by diminishing serum calcium concentrations. Subsequent studies have demonstrated that serum ionized calcium concentration are indeed lower in women with pre eclampsia than in matched normotensive controls.

Urinary calcium excretion is inversely related to the incidence of pre-eclampsia. Extracellular ionized calcium ion concentrations are crucial for the production of endothelial nitric oxide (NO) which is involved in regulation of vascular tone.

Rodiguez et al. ${ }^{(2)}$ studied 25 pregnant patients, of which 13 were normotensive and 12 were preeclamptic. He found that 24 hour calciuria was lower in pre eclampsia group than normotensive group.

Suarez et al. ${ }^{(3)}$ compared the calciuric response in symptom free primigravid women to an oral calcium load between those with normal urinary calcium excretion and those with relatively low urinary calcium excretion. There was a 5 fold difference in the response to calcium load between hypocalcuric women and the normocalciuric women. 
Suarez et al. ${ }^{(3)}$ studied 69 young primigravidas to assess the efficacy of calciuria as a diagnostic test for prediction of pre eclampsia. The most efficient test was found to be urinary excretion of calcium per 24 hours based on body weight using 3.4 $\mathrm{mg} / \mathrm{kg} / 24$ hours as a cut off point. They concluded that in primagravidas a low urinary calcium excretion / $\mathrm{kg}$ body weight/ 24 hours before the end of first half of gestation in a risk factor for pre eclampsia with an acceptable sensitivity and high negative predictive value, but with a positive predictive value no better than chance.

Ramos et al. ${ }^{(5)}$ compared urinary calcium excretion in chronic hypertension patients with those with pre eclampsia and in normotensive patients. Patients with pre eclampsia ( $82 \pm 15 \mathrm{mg} / 24 \mathrm{hrs}$ ) showed significantly lower calciuria $(\mathrm{p}<0.05)$ than the group with chronic hypertension $(147 \pm 24.9 \mathrm{mg} / 24$ hour $)$ and the normotensive group (317 $\pm 86 \mathrm{mg} / 24$ hour) $\mathrm{p}<0.05$. Calciuria was significantly lower in the group with pre eclampsia than in the chronic hypertension group.

XieY et al. ${ }^{(4)}$ studied 103 patients in their third trimester of pregnancy and the relation between the 24 hour calcium excretion and the calcium to creatinine ratio of a single void urine sample. The 24 hour calcium excretion in patients with pregnant hypertension syndrome was significantly lower $(0.70 \pm 0.36 \mathrm{mmol} / \mathrm{L})$ than that in normal pregnant women $(4.0 \pm 1.9 \mathrm{mmol} / \mathrm{L}) \mathrm{p}<0.0005$. The 24 hour calcium excretion correlated well with the calcium to creatinine ratio of a single void morning urine sample in the two groups. The 24 hour urinary excretion can be estimated from single void urine sample and urinary calcium excretion can be used as an indicator for pregnant hypertension syndrome.

Ramos et al. ${ }^{(6)}$ studied 24 hours urine data from 143 obstetric patients, 33 with pre eclampsia, 58 normotensive and 52 patients with gestational hypertension. Using receiver operator curve, a urine calcium threshold of $12 \mathrm{mg} / \mathrm{dl}$ was chosen as predictive for the development of preeclampsia with a sensitivity of $85 \%$ specificity of $91 \%$ and positive and negative values of $85 \%$ and $91 \%$ respectively. Urinary calcium levels below $12 \mathrm{mg} / \mathrm{dl}$ may help distinguish preeclampsia from other hypertensive disorders of pregnancy.

Barton et al. ${ }^{(6)}$ tried to study the effect of Nifedipine on urinary excretion of calcium in pre eclampsia. 148 women with mild pre eclampsia were randomly allocated to treatment with either bed rest alone or in combination with Nifedipine at 26-36 weeks gestation. Significant reduction in calcium excretion was noted following Nifedipine therapy (62 \pm 94 mg calcium / $24 \mathrm{hr}$ ) compared to the control group (143 $\pm 153 \mathrm{mg} \mathrm{Ca} / 24$ hour) $\mathrm{p}<0.001$. Hence, progressive hypocalciuria is a feature of pre eclampsia.

What causes hypocalciuria is not well understood. In pregnancy there is increase in renal plasma flow and glomerular filtration rate. Increase in renal plasma flow causes $50 \%$ increase in GFR to an average of $137 \mathrm{ml} / \mathrm{min}$. The creatinine clearance is increased leading to reduced serum creatinine level average being $0.6 \mathrm{mg} \%$

Reduced urinary calcium excretion may be the result of dietary, renal or hormonal factors. Urinary calcium exhibits a balance between glomerular filtration and tubular reabsorption. Parathormone and 1,25, $(\mathrm{OH}) 2$ Vitamin D may influence tubular reabsorption of calcium. Observations by Taufield et al. ${ }^{(11)}$ suggested that alterations in 1,25 (OH)2 Vitamin D are not responsible for hypocalciuria.

Studies have demonstrated that serum ionized calcium concentrations are indeed lower in women with pre eclampsia. Vasoconstriction resulting from low calcium causes decrease in GFR, increased tubular reabsorption of calcium and decreased urinary calcium. Hypocalciuria could be partly due to decrease in GFR in preeclampsia but tubular reabsorption was also reduced because the fractional excretion of calcium was decreased. Since parathormone and calcium levels were not altered, the difference in calcium metabolism was not due to alterations in these hormones.

Suarez et al. ${ }^{(3)}$ subjected preeclamptic women and normotensive women to a calcium load and assessed the urinary calcium excretion. Both the groups responded to calcium load indicating poor calcium excretion, therefore is not due to poor 
absorption of calcium and also hypocalciuria continued despite antihypertensive therapy.

Mean values of calcium excretion obtained in this study are lower than that in various studies. This may be due to dietary deficiency of calcium.

Attallah et al. ${ }^{(10)}$ studied the effect of calcium supplementation on maternal and child adverse outcomes and concluded that there was no overall effect on the risk of preterm delivery although a modest reduction in risk amongst women at high risk of hypertension.

Urinary calcium to creatinine ratio was observed to be low in pre eclampsia group in this study. This finding is in contrast to the studies of Raniolo et al. ${ }^{(8)}$ and Baker et al. ${ }^{(9)}$ This finding supports the studies of Suzuki et al. ${ }^{(7)}$ and Xie et al. ${ }^{(4)}$

Various studies have shown that calcium supplementation helped those with high risk to develop pregnancy induced hypertension especially in countries where the calcium intake is low.

Largest randomized trial of calcium by Levine et al involving 4589 healthy nulliparous women failed to demonstrate beneficial effect of calcium in preventing pregnancy induced hypertension, intra uterine growth retardation, preterm deliveries, fetal or neonatal deaths.

Although recent trials with calcium are discouraging there may still be a benefit to calcium supplementation in high-risk population.

\section{Conclusions}

The study confirms hypocalciuria and decreased urinary calcium to creatinine ratio occurs in pregnant women with pre eclampsia. The mechanism is not well established. The urine calcium to creatinine ratio was found to be helpful in predicting pre-eclampsia.

\section{References}

1. Norwitz, Errol R.; Robinson, Julian N.; Repke, J Prevention of Preeclampsia: Is It Possible?, J. Clinical Obstetrics and Gynaecology, 1999; 42 (3), 445-449

2. Rodriguez JG, Avendano R, Austral de Chile, Valdivia Braz Jr Med Biol Res.
Hypocalciuria in pre eclampsia (Article in Spanish) 1998; (4):519-22.

3. Suarez VR, Trelles JG, Miyahira JM. Urinary calcium in asymptomatic primigravida who later developed preeclampsia. J Obstet Gynecol. 1996 ; 87:79-82.

4. Xie Y, Zhang G Liu X, Qiu W, (Article in Chinese) Urinary calcium excretion in patients with pregnant hypertension syndrome Hua Hsi I Ko Ta Hseuh Hseuh Pao 1995 Mar; 26(1):94-7.

5. Ramos L. S, Sandroni S, Andrez FT Calcium excretion in preeclampsia Obstet Gynecol 1991 Apr;77(4):510-3

6. Barton JR, Mercer BM Sibai BM, The effect of Nifedipine on urinary excretion of calcium in pre eclampsia American journal of Perinatology 1997 Nov;14(10):609-12

7. Suzuki Y, Hayashi Y, Murakami I Urinary calcium excretion as an early prediction marker for pregnancy induced hypertension (Article in Japanese) Nippon Sanka Fujinka Gakkai Zasshi 1992 Nov, 44(11)1421-6

8. Raniolo E, Phillipou G Prediction of pregnancy induced hypertension by means of the urinary calcium to creatinine ratios Med J Aust 1993 Jan 18;158(2) 98-100.

9. Baker PN, Hackett GA, The use of urinary albumin creatinine ratio and calcium creatinine ratio as screening tests for pregnancy induced hypertension Obstet Gynecol 1994;83(5) 745-9

10. Attalah AN, Hofmeyr GJ Duley L Calcium supplementation during pregnancy for preventing hypertensive disorders and related problems Cochrane Database Syst Rev 2000;(3) CD001059.

11. Taufield P A, Ales K I, Resnick L,Druzin ML N,Hypocalciuria in pregnancy New Eng J med 1987;316:715718S. M. Metev and V. P. Veiko, Laser Assisted Microtechnology, 2nd ed., R. M. Osgood, Jr., Ed. Berlin, Germany: name of the journal, 1998. 